\title{
Design, development and in vivo pharmacokinetic evaluation of zotepine loaded solid lipid nanoparticles for enhanced oral bioavailability
}

\author{
Nagaraj BANALA*, Tirumalesh C, Dinesh SURAM, Narendar DUDHIPALA \\ * Nanotechnology and Novel Drug Delivery Laboratory, Department of Pharmaceutics, University College of Pharmaceuti- \\ cal Sciences, Kakatiya University, Warangal, Telangana-506009, India. \\ Running title: Zotepine lipid nanoparticles
}

\begin{abstract}
The purpose of this work was to prepare and evaluate the zotepine (ZT) loaded solid lipid nanoparticles (SLNs) that might improve the oral bioavailability. ZT-SLNs were developed using homogenization method and characterized for optimal system based on physicochemical characteristics and in-vitro release. Optimized ZT-SLNs were evaluated for permeation, crystalline nature using DSC and XRD, surface morphology using SEM and physical stability. Further, pharmacokinetic (PK) studies of ZT-SLN were conducted in Wistar rats comparison with ZT-coarse suspension (ZT-CS). Optimized formulation showed Z-avg, PDI, ZP of 138.1 $\pm 3.2,0.23 \pm 0.02$ and $-26.4 \pm 1.5 \mathrm{mV}$, respectively. In-vitro release studies showed prolonged release, DSC and XRD studies revealed the conversion of ZT to amorphous form. SEM studies showed spherical shape. Permeability and PK studies showed 1.4-folds and 2.o-folds improvement in oral bioavailability, respectively in comparison with ZTCS formulation. Therefore, the results concluded that SLNs could be considered as a new alternative delivery system for the enhancement of oral bioavailability of ZT.
\end{abstract}

Keywords: Zotepine, solid lipid nanoparticles, crystallinity, in vitro release, ex vivo permeation, oral bioavailability.

\footnotetext{
Correspondence: Dr. B. Nagaraj

Dept of Pharmaceutics,

University college of Pharmaceutical Sciences,

Kakatiya University, Warangal,

Telangana 506009, India.

Email: bnrpharmacy@kaktiya.ac.in; dnrku14@gmail.com

ORCIDs:

Nagaraj Banala, 0000-0001-6150-6716

Dinesh Suram, 0000-0001-9601-5976

Narendar Dudhipala, 0000-0002-0711-601X

(Received 02 January 2020, accepted 11 November 2020)
} 


\section{INTRODUCTION}

The amount of the drug reaching to the systemic circulation mainly depends on the rate of absorption, which in turn depends on the drug solubility ${ }^{1}$. Oral delivery of the drugs is the most conventional and widely used technique for the administration of majority of active moieties. However, several compounds are unsuccessful and failed owing to their low absorption and low bioavailability upon oral administration ${ }^{2}$. The drugs with poor oral bioavailability are unable to reach the minimum effective concentration to exhibit therapeutic action. Some of the reasons for poor bioavailability include: poor solubility; inappropriate partition coefficient as it influences the permeation of drug through lipid membrane; first-pass metabolism; P-glycoprotein (P-gp) mediated efflux; and degradation of drug in the gastrointestinal tract (GIT) due to $\mathrm{pH}$ of the stomach or enzymatic degradation or by chemical ${ }^{3}$.

To overcome the solubility and oral bioavailability problems of Biopharmaceutical classification system (BCS) class II and IV drugs, the research in the present scenario is focused on bioavailability improvement by using various solubility enhancement techniques like liquisolid compacts ${ }^{4,5}$, micronization using nanosuspensions ${ }^{6}$, solid dispersions using complexation ${ }^{7,8}$, salt formation 9 . Majority of the drugs undergo first-pass effect after oral administration, there by the therapeutic concentration required in systemic circulation is lowered. However, by using the lipid based delivery systems both the solubility and first-pass effect problems are resolved and subsequently oral absorption was improved ${ }^{3}$.

Lipid based drug delivery systems are one of the approaches, which favors the lymphatic transport of oral administered drugs by escaping the first-pass effect $^{10,11}$. Colloidal lipid nanocarriers such as solid lipid nanoparticles (SLNs) and nanostructured lipid carriers (NLCs) are the promising approaches in the lipid based delivery systems. SLNs are mainly developed with the solid lipid carrier, used for incorporation of drug, and lipid dispersion is stabilized by the use of biocompatible surfactants ${ }^{12}$. SLNs offer remarkable advantages such as low toxicity, high bioavailability (pharmacokinetic and pharmacodynamic) of drugs, versatility of incorporation of hydrophilic and lipophilic drugs, and feasibility of large-scale production.SLN formulations have controlled drug release properties and provides enhanced chemical stability of drug molecules ${ }^{13}$. SLNs also used for drug targeting to various tissues such as liver ${ }^{14}$ and ocular ${ }^{15}$ for treatment of various diseases.

Zotepine (ZT) is an atypical anti-psychotic drug, which belongs to BCS class II category. The mechanism of action of ZT is mediated through combined dopa- 
mine and serotonin antagonisms ${ }^{16}$. It has a poor oral bioavailability of about 7-13\%, due to poor aqueous solubility ( $0.046 \mu \mathrm{g} / \mathrm{L}$ ), highly lipophilic (log P 5.6), and also hepatic first-pass metabolism. The drug levels were also decreased in the presence of CYP enzyme ${ }^{17,18}$. In oral route, $30 \%$ of the drug metabolizes into Nor-zotepine (active metabolite) and remaining 70\% of drug transforms to inactive metabolites such as 3-hydroxyzotepine, 2-hydroxyzotepine and zotepine-S-oxide ${ }^{16}$. Previously, Pailla et al. ${ }^{19}$ developed intranasal delivery of ZT for enhanced brain targeting, using nanosuspension formulation. The enhanced solubility and in vitro dissolution rate of ZT were observed and reported with self-emulsifying drug delivery systems ${ }^{20}$. But, till now there are no lipid nanocarrier systems were reported for enhanced oral delivery of ZT. Hence, in this attempt ZT loaded SLNs were developed could might be improve the oral bioavailability.

The objective of the present investigation was aimed to develop and characterize ZT loaded SLNs for enhanced oral delivery. Accordingly, ZT-SLNs were prepared using know methods and evaluated for an optimized formulation based on the physical and chemical parameters, in vitro release studies. Further, optimized formulation also characterized for solid state characterization for crystallinity, SEM for surface morphology and ex vivo permeation studies. In vivo performance of optimized ZT-SLN were observed by pharmacokinetic (PK) studies in male Wistar rats, comparison with ZT coarse suspension (ZT-CS) as control.

\section{METHODOLOGY}

\section{Materials}

Zotepine was a kind gifted sample from Symed labs, Hyderabad, India. Stearic acid (SA), glyceryl monostearate (GMS), Dynasan-118, Dyansan-116 and Dynasan-114 were purchased from Sigma-Aldrich, Hyderabad, India. Compritol 888 ATO was gift sample from Neuheit Pharma Technologies Private Ltd., Hyderabad. Soylecithin was a gifted sample from Lipoid, Ludwigshafen, Germany. Captex355, Captex200 were gift samples from Abitec corporation. All other chemicals were of analytical grade and solvents were of HPLC grade.

\section{Methods}

\section{Solubility studies}

ZT solubility in various solid lipids (stearic acid, glyceryl monostearate (GMS), Compritol ATO 888, Precirol ATO5, Dyansan-114, Dyansan-116 and Dynasan-118) was determined by using shaking method. Solid lipids were heated to above $5^{\circ} \mathrm{C}$ of their melting points. An excess amount of ZT was added and was 
continuously stirred on gyrator shaker at $180 \mathrm{rpm}$ for $48 \mathrm{~h}$. The supernatant was collected and filtered through $0.45 \mu$ membrane filter after centrifugation ${ }^{21}$. Necessary dilutions were made to the filtrate with methanol and estimated the drug concentration using UV-Visible spectrophotometer (SL-159, Elico, Hyderabad, India) at $\lambda_{\max }$ of $261 \mathrm{~nm}$. Simultaneously, solubility of ZT in release media such as distilled water, $0.1 \mathrm{~N} \mathrm{HCl}(\mathrm{pH}$ 1.2), phosphate buffer of $\mathrm{pH} 6.8$ and $\mathrm{p} \mathrm{H} 7.4$.

\section{Preparation of solid lipid nano particles (SLNs)}

ZT-SLNs were prepared using homogenization-probe sonication method, based on film hydration method ${ }^{22}$. Required amounts of ZT, solid lipid (SA, GMS, Compritol ATO888), and soy lecithin were dissolved in $10 \mathrm{~mL}$ mixture of chloroform and methanol (1:1) in a round bottom flask. Organic solvents were removed using rota evaporator (Heidolph, Germany). Drug embedded lipid layer was molten by heating at $5{ }^{\circ} \mathrm{C}$ above the melting point of the solid lipid. Aqueous phase was prepared by dissolving Poloxamer188 in double distilled water and heated to the same temperature of oil phase. Hot aqueous phase was added to oil phase. Homogenization was carried out at 12,000 rpm for $5 \mathrm{mins}$ to form pre-emulsion. The obtained emulsion was ultra-sonicated using Probe sonicator with $12 \mathrm{~T}$ probe tip (Bandelin, Germany) for 20 mins. ZT-SLNs were obtained upon cooling to room temperature. The composition of the SLNs are given in Table 1.

Table 1: Composition of zotepine loaded solid lipid nanoparticles and control formulation

\begin{tabular}{|c|c|c|c|c|c|c|c|}
\hline Ingredients (\%w/v) & ZT-SLN1 & ZT-SLN2 & ZT-SLN3 & ZT-SLN4 & ZT-SLN5 & ZT-SLN6 & ZT-CS \\
\hline Zotepine (mg) & 0.1 & 0.1 & 0.1 & 0.1 & 0.1 & 0.1 & 0.1 \\
\hline Glyceryl monostearate & 1.0 & - & - & 2.0 & - & - & - \\
\hline Stearic acid & - & 1.0 & - & - & 2.0 & - & - \\
\hline Compritol AT0 888 & - & - & 1.0 & - & - & 2.0 & - \\
\hline Soylecithin & 1.0 & 1.0 & 1.0 & 1.0 & 1.0 & 1.0 & - \\
\hline Poloxamer 188 & 1.5 & 1.5 & 1.5 & 1.5 & 1.5 & 1.5 & - \\
\hline Na CMC & - & - & - & - & - & - & 0.05 \\
\hline Water (mL) & QS 10 & QS 10 & QS 10 & QS 10 & QS 10 & QS 10 & QS 10 \\
\hline
\end{tabular}

* $\mathrm{Na} \mathrm{CMC}$ - sodium carboxy methyl cellulose 


\section{Preparation of ZT coarse suspension (ZT-CS)}

ZT-CS formulation was prepared by taking about $100 \mathrm{mg}$ of sodium carboxy methyl cellulose (suspending agent) in a mortar and triturated for 15 mins. Then, $10 \mathrm{mg}$ of ZT was added to it and together triturated. To it, $10 \mathrm{~mL}$ of double distilled water was added and again triturated for about $5 \mathrm{~min}$ to obtain ZT-CS $(1 \mathrm{mg} / \mathrm{mL})$. This was used as control formulation.

\section{Characterization of ZT-SLNs}

\section{Measurement of particle size, PDI and zeta potential (ZP)}

The mean size as Z-avg (nm), PDI and ZP of ZT-SLNs were measured by using photon correlation spectroscopy, using a Malvern Zetasizer (Nano ZS9o, UK). About $100 \mu \mathrm{L}$ of prepared SLN formulations were diluted to $5 \mathrm{~mL}$ with double distilled water to get optimum Kilo Counts Per Second (Kcps) of 50-200 for measurements in triplicate at $25^{\circ} \mathrm{C}^{23}$.

\section{Drug content and entrapment efficiency (EE)}

About $0.1 \mathrm{~mL}$ of prepared ZT-SLN formulation was taken and diluted with chloroform: methanol (1:1) and drug content of the diluted samples was estimated by HPLC-method. The EE (\%) in ZT-SLN formulation was calculated by estimating the concentration of the free ZT in the aqueous phase of an undiluted formulation, using an ultrafiltration method centrisort tubes (Sartorius, Germany). The aqueous medium was separated by ultra-filtration using centrisort tubes (Sartorius, Germany) which consisting a filter membrane (M.Wt.20,000 Daltons) at the base of the sample recovery chamber. Centrifugation was carried at $5000 \mathrm{rpm}$ for $30 \mathrm{~min}^{24}$. The SLN along with the encapsulated drug remained in outer chamber and aqueous phase moved into sample recovery chamber through filter membrane. The amount of zotepine in aqueous phase was estimated by HPLC method. The EE was calculated by using the equation 1:

$\% \mathrm{EE}=\left[\frac{\mathrm{Di} \_\mathrm{Df}}{\mathrm{Di}}\right] \times 100 \quad(1)$

Where, $\mathrm{D}_{\mathrm{i}}$ is the total ZT content and $\mathrm{D}_{\mathrm{f}}$ is the free ZT present in the aqueous phase. 


\section{In vitro drug release studies}

The in vitro drug release study of ZT-SLN and ZT-CS formulation was performed using dialysis method. Dialysis membrane (Hi media, Hyderabad, India) having average pore size $2.4 \mathrm{~nm}$ and molecular weight cut-off between12,000-14,000 Daltons were used for the release studies ${ }^{25}$. Dialysis membrane was soaked overnight in double distilled water prior to the study. Release studies were carried out for first $2 \mathrm{~h}$ in $0.1 \mathrm{~N} \mathrm{HCl}$, followed by pH6.8 phosphate buffer for $48 \mathrm{~h}$ by open tube method. Dialysis membrane was tied to open tube which acts as a donor compartment. Temperature of receptor compartment was maintained at $37 \pm 0.5^{\circ} \mathrm{C}$. Two $\mathrm{mL}$ sample was withdrawn at regular time points of $0,0.25,0.5$, $1,2,3,4,6,8,10,12,24,36$, and $48 \mathrm{~h}$ from receiver compartment and replenished with same volume of fresh buffer. The collected samples were suitably diluted and analyzed by UV-Visible spectrophotometer.

\section{Ex vivo studies by normal sac method}

Ex vivo studies were performed for optimized ZT-SLN formulation by using evert-sac method ${ }^{26}$. The studies were conducted with prior approval of Institutional Animal Ethical Committee (IAEC/o1/UCPSC/KU/2018). Evert-sac studies were carried out in order to investigate the permeation behavior of the control formulation and optimized ZT-SLN formulation ( $1 \mathrm{~mL}$ ) across the small intestine. In this study, male Wistar rats of $200 \pm 30 \mathrm{~g}(\mathrm{n}=3)$ were taken and subjected to overnight fasting. The rats were sacrificed by cervical dislocation technique and jejunum of $4 \mathrm{~cm}$ were isolated, flushed with saline solution and transferred into oxygenated Krebs's ringer solution. The one end of the sac was tied with thread and filled with the optimized ZT-SLN or ZT-CS as control (1 $\mathrm{mL}$ ) formulations and the other end was tied. The segment was immersed in $100 \mathrm{~mL}$ in Krebs's ringer solution and the medium was oxygenated using aerator. At regular time intervals (o, 15, 30, 45, 60, 90 and $120 \mathrm{~min})$ samples were withdrawn from beaker and analyzed for drug content ${ }^{27}$. The permeability coefficient of the SLN and control formulation was calculated from the slope of linear portion, by plotting the percentage drug permeated vs time ${ }^{28}$.

\section{Lyophilization of ZT-SLNs}

The optimized ZT-SLN formulation was subjected to lyophilization. The SLN formulation was prepared, using $10 \% \mathrm{w} / \mathrm{w}$ of trehalose dihydrate as cryoprotectant. Prepared ZT-SLN formulation was kept in $-80^{\circ} \mathrm{C}$ freezer for overnight and subjected to lyophilization (Lyodel, Chennai, India) with applied vacuum ${ }^{29}$, ${ }^{30}$. Lyophilized formulation was subjected to particle size analysis, drug content, $\mathrm{EE}$ and in vitro release before and after lyophilization. 


\section{Solid-state characterization}

\section{Differential Scanning Calorimetry (DSC)}

DSC experiments were through using Perkin Elmer DSC 4000 (USA) model to find out the presence of any interaction between drug and the excipients and also to check the alterations in crystallinity of the drug. About $8 \mathrm{mg}$ of the pure drug, physical mixture and optimized lyophilized ZT-SLN formulation samples were taken in aluminium pans, using dry nitrogen as purging gas. The heating rate was $10^{\circ} \mathrm{C} / \mathrm{min}$ and the obtained thermograms were observed for any type of interaction ${ }^{31}$.

\section{$X$-ray diffraction studies}

Crystalline nature of Powder X-ray diffractometer (XRD-60oo, Shimazdu, Japan) was used for diffraction studies (Arun et al., 2017). Powder XRD studies were performed on the samples by exposing them to nickel filtered $\mathrm{CuKa}$ radiation ( $40 \mathrm{kV}, 30 \mathrm{~mA}$ ) and scanned from $2^{\circ}$ to $70^{\circ}, 2 \theta$ at a step size of $0.045^{\circ}$ and step time of $0.5 \mathrm{~s}$. Samples used for PXRD analysis were pure ZT, pure lipid and lyophilized ZT loaded solid lipid nanoparticles ${ }^{32}$.

\section{Surface morphology by scanning electron microscopy (SEM)}

The morphology of pure ZT and freeze-dried ZT-SLN formulation were investigated by scanning electron microscope (SEM S-3700, Hitachi, Japan). The samples were fixed on a brass stub and were coated by thin layer of gold to make electrically conductive ${ }^{25}$. SEM images were recorded at various magnification levels.

\section{Bioavailability study}

\section{Study design and sampling schedule}

Healthy male Wistar rats (weighing $210 \pm 30 \mathrm{~g}$ ) were used for the PK study. The animals were fasted overnight and had free access to water. The studies were conducted with prior approval of Institutional Animal Ethical Committee (IAEC/01/UCPSC / KU/2018). The animals were divided into two groups (each with $\mathrm{n}=6$ ) and were orally administered with ZT-CS and optimized ZT-SLN formulation at a dose of $2.2 \mathrm{mg} / \mathrm{kg}$ body weight. Both the formulations were administered orally with the help of sterilized rat oral feeding tube. At predetermined time intervals, $0.5 \mathrm{~mL}$ of blood samples were collected at predetermined (o, $0.5,1,2,4,6,8,10,12,24$ and $48 \mathrm{~h}$ ) time intervals by retro-orbital venous plexus puncture. The blood samples were allowed to clot and centrifuged for 15 mins at $3000 \mathrm{rpm}$. The serum was separated and transferred into clean micro centrifuge tubes and stored at $-20^{\circ} \mathrm{C}$ until analysis. 


\section{HPLC method}

HPLC analysis was conducted with $\mathrm{C}_{18}$ column (250 mm X $4.6 \mathrm{~mm} ; 5 \mu \mathrm{m}$ ). Mobile phase consisting of 45: $55 \mathrm{v} / \mathrm{v}$ ratio of acetonitrile and phosphate buffer adjusted to $\mathrm{pH} 4.7$ at a flow rate of $1 \mathrm{~mL} / \mathrm{min}$ at $261 \mathrm{~nm}$ with $\mathrm{UV}$ detector was used for detection of peaks.

\section{Extraction procedure from rat serum sample}

To $100 \mu \mathrm{L}$ of serum, $100 \mu \mathrm{L}$ of internal standard (Ramipril, $2 \mu \mathrm{g} / \mathrm{mL}$ ), $300 \mu \mathrm{L}$ of methanol was added. After vortex mixing for $5 \mathrm{~min}$ at room temperature, the samples were centrifuged at $5000 \mathrm{rpm}$ for $15 \mathrm{~min}$. After centrifugation the whole organic layer was separated and transferred into another eppendorf tube. Finally, a volume of $20 \mu \mathrm{L}$ was injected into HPLC system.

\section{Estimation of Pharmacokinetic parameters and statistical significance}

Kinetica 2000 software (version 5.0, USA) was used for the estimation of PK parameters like Cmax, Tmax, AUC, $\mathrm{t}_{1 / 2}$ and MRT and the values were expressed in mean $\pm \mathrm{SD}$. One-way ANOVA was performed using Graph pad prism software (version 5.03, San Diego, CA, USA) for statistical comparison of data at $p$ value of less than 0.05 .

\section{Physical stability studies}

An optimized ZT-SLN formulation was stored at room temperature and refrigerated temperature conditions over two months to assess the physical and chemical stability. Z-avg, PDI, ZP, assay and EE of the samples were observed at predetermined time intervals and checked for statistically significance.

\section{RESULTS AND DISCUSSION}

\section{Solubility studies}

Solubility of ZT was studied in various solid lipids. SA, GMS, Compritol ATO 888 were selected as solid lipids for SLN formulations development. In order to determine the effect of mono and mixed glycerides on the SLN formulation SA, GMS and Compritol ATO 888 was selected. Order of solubility of ZT in solid lipids were GMS, SA, Dynasan-118, Dynasan-114 (showed nearly same solubility)>Compritol ATO888 > Dynasan-112 >Precirol ATO5. The solubility of ZT was decreased with increased $\mathrm{pH}$ of dissolution media and indicates $\mathrm{pH}$ dependent solubility. The order of solubility in release media as follows: water $>0.1 \mathrm{~N} \mathrm{HCl}>\mathrm{pH} 6.8$ phosphate buffer $>\mathrm{pH} 7.4$ phosphate buffer. 


\section{Characterization of ZT-SLNs}

ZT-SLNs formulations were prepared using three different lipids such as GMS, SA and Compritol ATO 888, each at 1 and $2 \% \mathrm{w} / \mathrm{v}$, respectively. Homogenization followed by probe sonication method used for the preparation of ZT-SLNs. The homogenization time and probe sonication time used at $12000 \mathrm{rpm}$ for 5 mins and 20 mins at $40 \%$ amplitude, respectively. The conditions were optimized based on the earlier reported methods ${ }^{23}$.

\section{Measurement of particle size, PDI and ZP}

Prepared ZT-SLNs were characterized for particle size, PDI and ZP, using Zetasizer and reported in Table 2. Particle size, PDI and ZP of the ZT-SLNs formulations (ZT-SLN1 to ZT-SLN6) were ranged from $137.3 \pm 3.5$ to $340.4 \pm 3.0$ $\mathrm{nm}, 0.22 \pm 0.01$ to $0.54 \pm 0.08,-10.6 \pm 0.3$ to $-27.4 \pm 2.5 \mathrm{mV}$, respectively. From the results, as the concentration of lipids increased from $1 \% \mathrm{w} / \mathrm{v}$ to $2 \% \mathrm{w} / \mathrm{v}$, increased in particle size and PDI, decreased in ZP were observed. This might be due to the changes in the orientation of the drug and presence of the void spaces of lipid and drug. PDI of the formulations (SLN1-SLN5) were below 0.5, except for formulations prepared with Compritol ATO $\AA 888$. The PDI value of up to 0.5 were considered to be homogeneous dispersion and was evidenced from the earlier reports. ${ }^{[15]} \mathrm{ZP}$ of the ZT-SLNs were crucial factor the stability assessment. In this case, the combination of surfactants imparts the electrostatic repulsion and steric stabilization as well. The presence of Poloxamer 188 in the development of SLNs could be favored for the maintenance of this $\mathrm{ZP}^{33}$. In general, $\mathrm{ZP}$ with $\pm 30 \mathrm{mV}$ could be considered as stable dispersion system. Formulations prepared with GMS showed above $-25 \mathrm{mV}$ and indicates stability. But, formulations prepared with SA had below $-20 \mathrm{mV}$ of $\mathrm{ZP}$.

Table 2: Physicochemical characteristics - size, PDI, Zeta potential, entrapment efficiency and assay of zotepine loaded solid lipid nanoparticle formulations (mean $\pm S D, n=3$ )

\begin{tabular}{|c|c|c|c|c|c|}
\hline Formulation & Size $(\mathbf{n m})$ & PDI & ZP $(\mathbf{m V})$ & Assay $(\mathbf{m g})$ & EE $(\%)$ \\
\hline ZT-SLN1 & $137.3 \pm 3.5$ & $0.22 \pm 0.01$ & $-26.7 \pm 1.7$ & $9.9 \pm 0.4$ & $95.1 \pm 2.1$ \\
\hline ZT-SLN2 & $154.5 \pm 4.7$ & $0.26 \pm 0.06$ & $-27.4 \pm 2.5$ & $9.2 \pm 0.4$ & $90.4 \pm 3.2$ \\
\hline ZT-SLN3 & $187.3 \pm 2.8$ & $0.46 \pm 0.05$ & $-10.6 \pm 0.3$ & $9.1 \pm 0.5$ & $85.7 \pm 2.6$ \\
\hline ZT-SLN4 & $253.7 \pm 5.8$ & $0.34 \pm 0.03$ & $-18.4 \pm 2.4$ & $8.7 \pm 0.4$ & $88.9 \pm 2.5$ \\
\hline ZT-SLN5 & $340.4 \pm 3.0$ & $0.29 \pm 0.07$ & $-29.4 \pm 2.1$ & $8.9 \pm 0.1$ & $92.6 \pm 1.6$ \\
\hline ZT-SLN6 & $303.3 \pm 2.1$ & $0.54 \pm 0.08$ & $-25.6 \pm 1.0$ & $8.8 \pm 0.4$ & $94.8 \pm 3.5$ \\
\hline
\end{tabular}




\section{Drug content and entrapment efficiency}

All the formulations were analyzed for EE and total drug content by HPLC (Table 2). EE of the all the formulations (ZT-SLN1 to ZT-SLN6) were ranged from $85.7 \pm 2.6$ to $95.1 \pm 2.1 \%$. As the concentration of lipid increases, EE of the ZTSLNs were decreased, but not significant difference. This is due to the presence of less orientation of drug molecule in the lipid matrix. Among all the developed formulations ZT-SLN1 formulation is having the high EE. Drug content of all the formulations were in the range of $8.7 \pm 0.4$ to $9.9 \pm 0.4 \mathrm{mg}$.

\section{In vitro dissolution studies}

All the prepared SLNs were subjected to in vitro release studies, using dialysis method. $0.1 \mathrm{~N} \mathrm{HCl} \mathrm{pH} 1.2$ followed by $\mathrm{pH} 6.8$ phosphate buffer for the release studies. In vitro release of ZT from SLN formulations were observed to $69 \pm 2.1$ to $82.9 \pm 3.6 \%$ range, over a $48 \mathrm{~h}$ (Figure 1). However, formulations prepared with $1 \%$ and $2 \% \mathrm{w} / \mathrm{v}$ lipid concentrations were not significantly influence the drug release behavior from the SLN formulations. This could be due to the high EE (more than 90\%) and also free drug availability in the outer lipid phase was very less. Formulations prepared with GMS, SA and Compritol ATO888 showed $83.3 \pm 2.4$ and $75.7 \pm 3.2 \%$ (from SLN1 and SLN2), $76.8 \pm 1.9$ and $73.6 \pm 2.3 \%$ (from SLN3 and SLN4), $71.5 \pm 1.2 \%$ and $68.5 \pm 1.8 \%$ (from SLN5 and SLN6), respectively. The crystal lattice of the lipid matrix might be the reason for difference in the drug release from ZT-SLNs. Monoglyceride formulations (GMS and SA) showed slightly better release then mixed glyceride (Compritol ATO®888) formulations. The drug release from the ZT-CS formulation showed $50.2 \pm 2.2 \%$ in $120 \mathrm{~min}$ (data not showed). In all the prepared formulations, ZT-SLN1 formulation prepared with GMS was having less particle size and PDI, stable ZP, with high $\mathrm{EE}$, acceptable drug content and also showed maximum drug release in $48 \mathrm{~h}$, comparison with other formulations. Therefore, ZT-SLN1 formulation was considered as an optimized formulation and selected for further studies. 


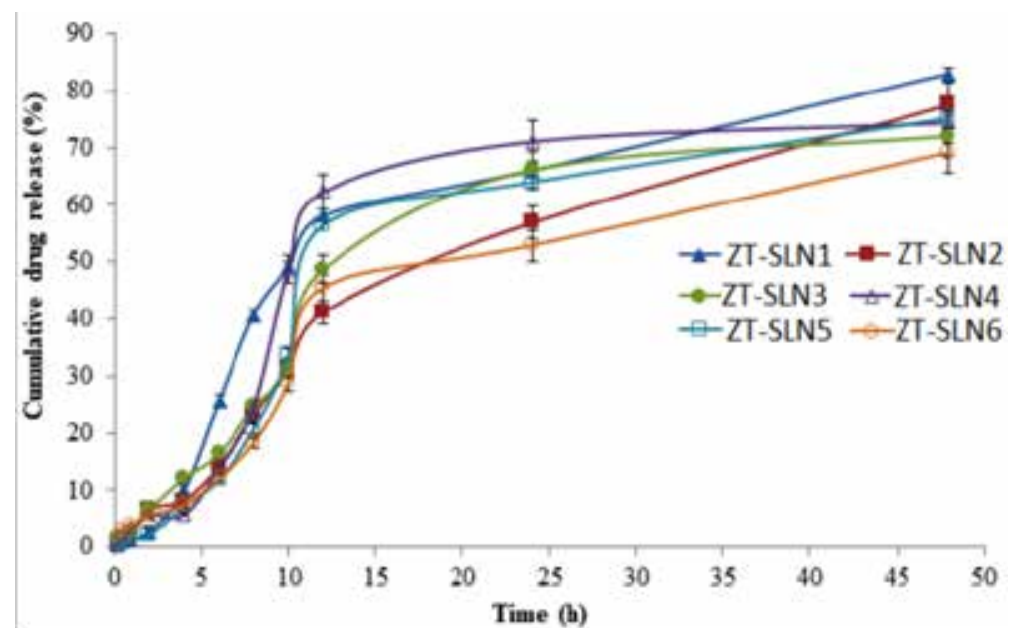

Figure 1: In vitro release profiles of zotepine from ZT-SLNs (mean $\pm S D, n=6$ )

\section{Ex vivo permeation studies}

Ex vivo permeation studies were performed for optimized ZT-SLN1 formulation in comparison with ZT-CS formulation through rat intestine using everted sac perfusion method. The duration of the study was $120 \mathrm{~min}$ and represented in Figure 2. From the results, the \% of ZT permeation from ZT-SLN1 and ZT-CS formulation was $18.8 \pm 1.6 \%$ and $28.3 \pm 2.2 \%$, respectively. Statistically significance difference $(\mathrm{p}<0.05$ ) was observed from the ZT-SLN1 compared with ZT-CS formulation. The prolonged release of the ZT from the ZT-SLN formulation was observed from the in vitro release and ex vivo permeation studies. Furthermore, the permeability rate coefficient of the ZT-SLN1 formulation was calculated from the slope of linear portion by plotting percentage drug permeation against time. From the results, permeability rate of the ZT-SLN1 and ZT-CS formulation was 0.19 and $0.13 \mathrm{ml} / \mathrm{min} . \mathrm{cm}^{2}$, respectively. About 1.4-folds enhancement in the permeation of ZT-SLN1 was observed compared with ZT-CS formulation. 


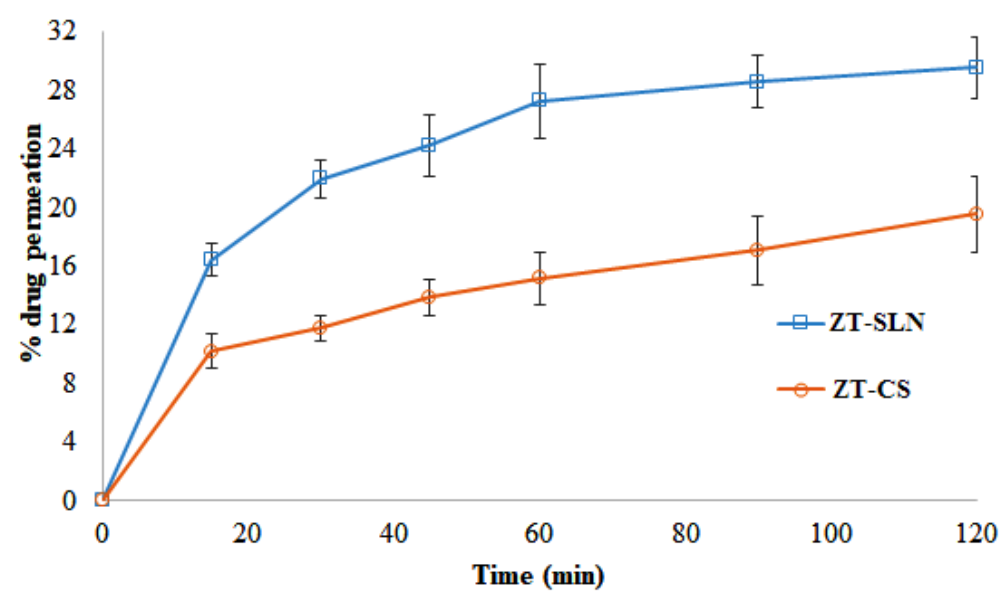

Figure 2: Ex vivo permeation profiles of zotepine from ZT-CS and ZT-SLN1 formulations $($ mean $\pm \mathrm{SD}, \mathrm{n}=3$ )

\section{Lyophilization of ZT-SLN}

The crystalline nature, surface morphology and conversion of lipid nanoparticles into solid dosage forms by incorporating in capsules or compression to tablets were easy by the conversion of dispersion to solid form of the nanoparticles. This is generally achieved with either spray drying or freeze drying techniques. Optimized ZT-SLN formulation was subject to lyophilization, using $10 \% \mathrm{w} / \mathrm{w}$ of trehalose as cryoprotectant. The cryoprotectant and its concentration were selected based on the previously reported methods ${ }^{24}$. The lyophilized formulation was characterized for before and after lyophilization and presented in Table 3. From the results, about two to three-fold increase in the particle size and PDI of the formulation was observed after lyophilization. This could be due to the aggregation of the particles during the process of freeze-drying. But, there were no significant changes noticed in the other parameters.

Table 3: Physicochemical characteristics of optimized ZT-SLN formulation before and after lyophilization (mean $\pm S D, n=3$ )

\begin{tabular}{|c|c|c|c|c|c|}
\hline Condition & Size (nm) & PDI & ZP (mV) & Assay (mg) & EE (\%) \\
\hline Pre--lyo & $145.2 \pm 2.7$ & $0.23 \pm 0.05$ & $-27.6 \pm 1.4$ & $9.8 \pm 0.3$ & $94.4 \pm 2.8$ \\
\hline Post-lyo & $288.9 \pm 5.2$ & $0.38 \pm 0.06$ & $-29.1 \pm 3.5$ & $9.9 \pm 0.6$ & $93.6 \pm 1.6$ \\
\hline
\end{tabular}




\section{Solid state characterization}

\section{Differential Scanning Calorimetry}

DSC studies were used to determine the compatibility status of the solid lipids and other excipients used in the SLN formulation, crystalline nature of the drug in the nanoformulations and was based on the fact that different lipids possessed different melting points and enthalpies. DSC thermograms of pure ZT, pure GMS, physical mixture of ZT and GMS in 1:1 ratio and lyophilized ZTSLN1 formulation are shown in Figure 3.

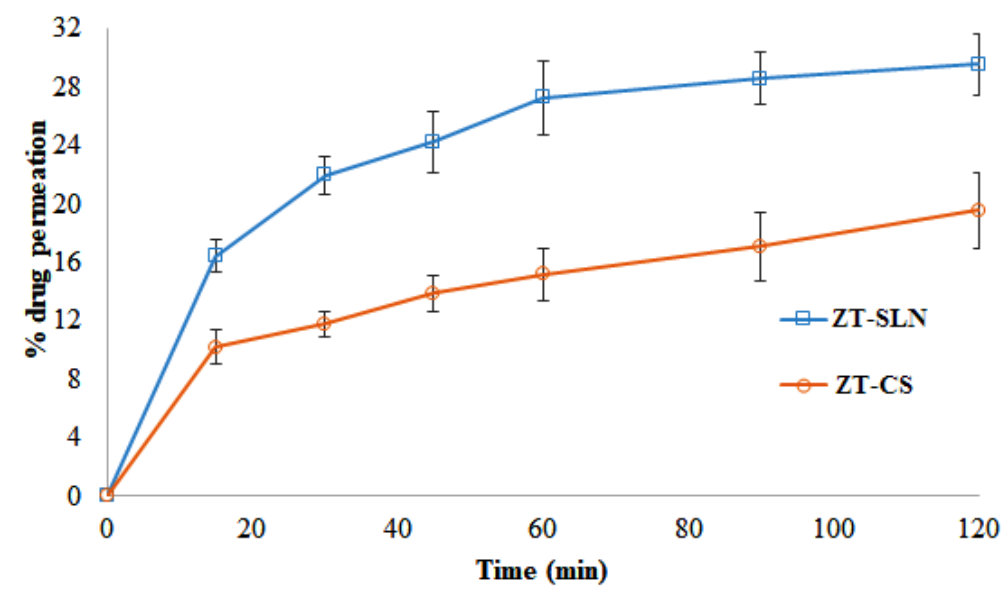

Figure 3: DSC thermograms of pure ZT (A), pure Dynasan-118 (B), physical mixture of ZT + lipid (1:1) (C) and lyophilized optimized ZT-SLN1 formulation (D)

The DSC thermogram of pure ZT showed a sharp endothermic peak at $97.76^{\circ} \mathrm{C}$ and it is corresponding to reported melting point. Pure GMS showed a sharp endothermic peak at $72.06{ }^{\circ} \mathrm{C}$. Physical mixture of ZT with GMS showed drug peaks at $98.82{ }^{\circ} \mathrm{C}$, however with less enthalpy compared with pure ZT enthalpy. But, melting endotherm of drug was well preserved with slight changes in terms of shifting in the temperature of the melt. It is known that the quantity of material used, especially in drug-excipient mixtures, could influence the peak shape and enthalpy. Thus, these minor changes in the melting endotherm of drug could be due to the mixing of drug and excipient, which lowered the purity of each component in the mixture and this, might not necessarily indicate potential incompatibility. The absence of endotherm peak of drug in lyophilized ZT-SLN1 formulation unravels the conversion of native crystalline state of the drug to amorphous state. 


\section{XRD studies}

XRD studies of pure ZT, pure lipid and lyophilized ZT-SLN1 were showed in Figure 4. From the results, powder-XRD patterns of ZT showed sharp peaks at $2 \theta$ scattered angles of 10.3, 11.4, 13.5, 17.1, 18.3, 19.8, 20.3, 23.2 and 24.1 degrees, these were indicating the crystalline nature of drug. These characteristic peaks of ZT peaks were absent in the lyophilized ZT-SLN1 sample. This specified that the ZT was not in crystalline form and converted to amorphous nature, after lyophilization of optimized ZT-SLN1. This reduction in crystallinity or conversion to amorphous state of ZT from ZT-SLN was noticed in DSC analysis as well ${ }^{34}$.

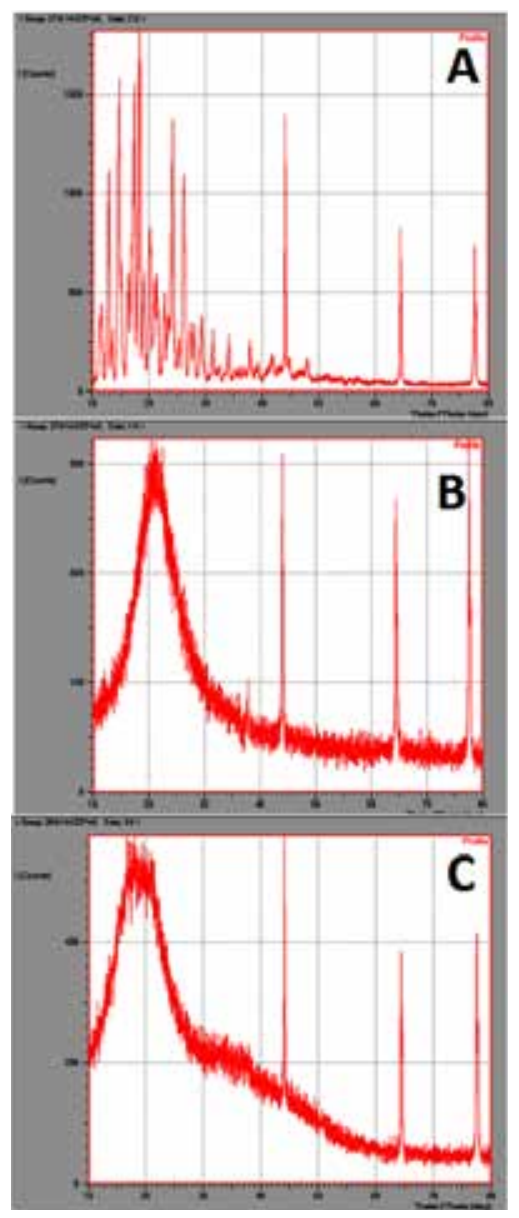

Figure 4: XRD spectra of pure ZT (A), pure Dynasan-118 (B), p and lyophilized optimized ZT-SLN1 formulation (C) 


\section{Scanning electron microscopy}

The shape of the ZT before and after loading onto SLN formulation was observed using SEM method. For this purpose, surface morphology study was conducted for pure drug and lyophilized ZT-SLN formulation. The results are showed in Figure 5. SEM picture revealed that pure drug of ZT particles are possessed different shaped (cubic and nearly spherical) but highly aggregated (Figure 5A). Optimized formulation of ZT-SLN1 particles are nearly spherical shaped and also aggregated ${ }^{35}$. The results of the SEM studies also correlated with particle size of after lyophilized sample.
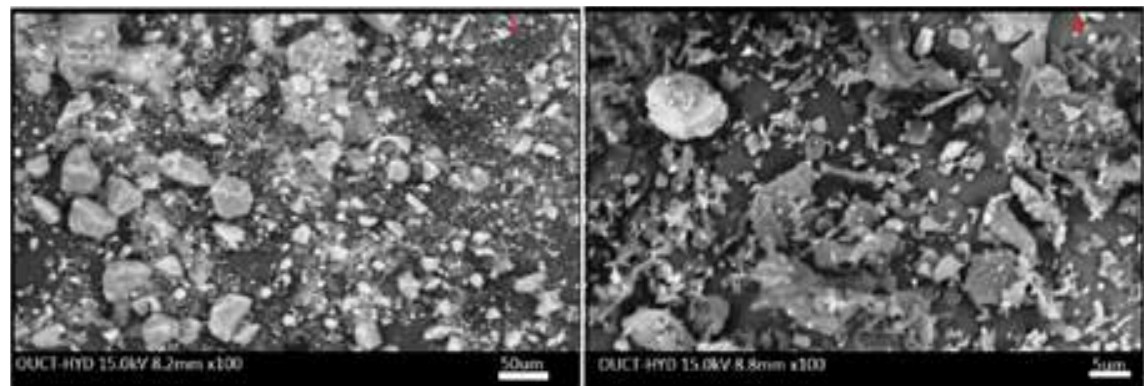

Figure 5: SEM images of pure ZT (A) and optimized ZT-SLN formulation (B)

\section{Stability study of optimized ZT-SLN}

The stability of the optimized ZT-SLN was monitored by storage at room temperature $\left(25^{\circ} \mathrm{C}\right)$ and refrigerated temp $\left(4^{\circ} \mathrm{C}\right)$ for 60 days. The formulations were analyzed on $1^{\text {st }}$ day, $30^{\text {th }}$ day, and $60^{\text {th }}$ day respectively for size, PDI, ZP, EE and drug content. The results indicated that no significant variations were noticed in all the measured parameters, and found to be stable up to two months (Table 4).

Table 4: Stability studies of optimized formulation of ZT-SLN (F1) at both room temperature $\left(25^{\circ} \mathrm{C}\right.$ ) and refrigerated $\left(4^{\circ} \mathrm{C}\right)$ for a period two of months (mean $\pm \mathrm{SD}, \mathrm{n}=3$ )

\begin{tabular}{|c|c|c|c|c|c|c|c|c|c|c|}
\hline \multirow{2}{*}{$\begin{array}{c}\text { Time } \\
\text { (day) }\end{array}$} & \multicolumn{5}{|c|}{ At room temperature $\left(25^{\circ} \mathrm{C}\right)$} & \multicolumn{5}{c|}{ At refrigerated temperature $\left(4^{\circ} \mathrm{C}\right)$} \\
\cline { 2 - 11 } & Size $(\mathrm{nm})$ & PDI & ZP & EE & Assay & Size $(\mathrm{nm})$ & PDI & potential & EE & Assay \\
\hline 1 & $138.1 \pm 3.2$ & $0.23 \pm 0.02$ & $-26.4 \pm 1.5$ & $9.9 \pm 0.3$ & $94.7 \pm 3.1$ & $138.1 \pm 3.2$ & $0.23 \pm 0.02$ & $-26.4 \pm 1.5$ & $9.9 \pm 0.3$ & $94.7 \pm 3.1$ \\
\hline 30 & $140.5 \pm 2.7$ & $0.23 \pm 0.04$ & $-27.1 \pm 1.0$ & $9.8 \pm 0.4$ & $92.3 \pm 2.4$ & $145.5 \pm 4.2$ & $0.24 \pm 0.06$ & $-25.4 \pm 1.9$ & $9.8 \pm 0.5$ & $91.4 \pm 2.8$ \\
\hline $\mathbf{6 0}$ & $144.3 \pm 2.7$ & $0.24 \pm 0.05$ & $-6.2 \pm 2.3$ & $9.8 \pm 0.6$ & $91.7 \pm 2.5$ & $148.3 \pm 5.3$ & $0.25 \pm 0.03$ & $-24.3 \pm 1.4$ & $9.7 \pm 0.4$ & $90.2 \pm 2.4$ \\
\hline
\end{tabular}




\section{PK study}

Oral bioavailability study for ZT-CS and optimized ZT-SLN1 formulation was performed in male Wistar rats with $n=6$ in each group. The collected serum samples were analyzed for drug content, using HPLC method. Ramipril is used as internal standard. Retention times of both ZT and IS were 7.8 and $4.5 \mathrm{~min}$, respectively. The linearity of the ZT was observed in the concentration range of $0.25-12 \mu \mathrm{g} / \mathrm{mL}$. The limit of detection and limit of quantification of the ZT were 0.1 and $0.2 \mu \mathrm{g} / \mathrm{mL}$, respectively. About $98.5 \%$ of extraction efficiency was observed from recovery studies.

The PK parameters of ZT from testing formulations were calculated using Kinetica 2000 software with non-compartmental model. Mean serum concentration vs time profiles of ZT-CS and ZT-SLN formulation after oral administration of single dose are shown in Figure 6. PK parameters like $\mathrm{AUC}_{\text {total }}, \mathrm{C}_{\max }, \mathrm{T}_{\max }$, MRT and $t_{1 / 2}$ of the formulations were calculated and shown in Table 5 . A significant difference observed in $\mathrm{C}_{\max }$ and $\mathrm{MRT}$ of the $\mathrm{F} 1$ formulation, indicates the prolonged release of the ZT. AUC is the major indicator of the bioavailability of the formulations. From the results, AUC values of ZT-CS and ZT-SLN1 formulations were found to be $18.2 \pm 1.3$ and $37.1 \pm 2.2 \mu \mathrm{g} / \mathrm{mL} . \mathrm{h}$, respectively and were statistically significant ( $\mathrm{p}<0.05$ ). From the results, about 2.0-folds improvement in the bioavailability observed from ZT-SLN formulation in comparison with ZT-CS formulation. The improved oral bioavailability of SLNs of ZT could be due to the contribution of individual and/or combined effects of nanosized particles of the SLNs. The effective surface would influence the adhesion to GIT. Consequently, there is increased contact time of the SLN particles ${ }^{36-42}$. In addition, the soylecithin and poloxamer could alter the permeability characters of the GI membrane. The fatty acid chains present in the lipids of SLNs improve the uptake by lymphatic transport. This lymphatic transport minimizes the first-pass effect of the drug ${ }^{43-47}$. 


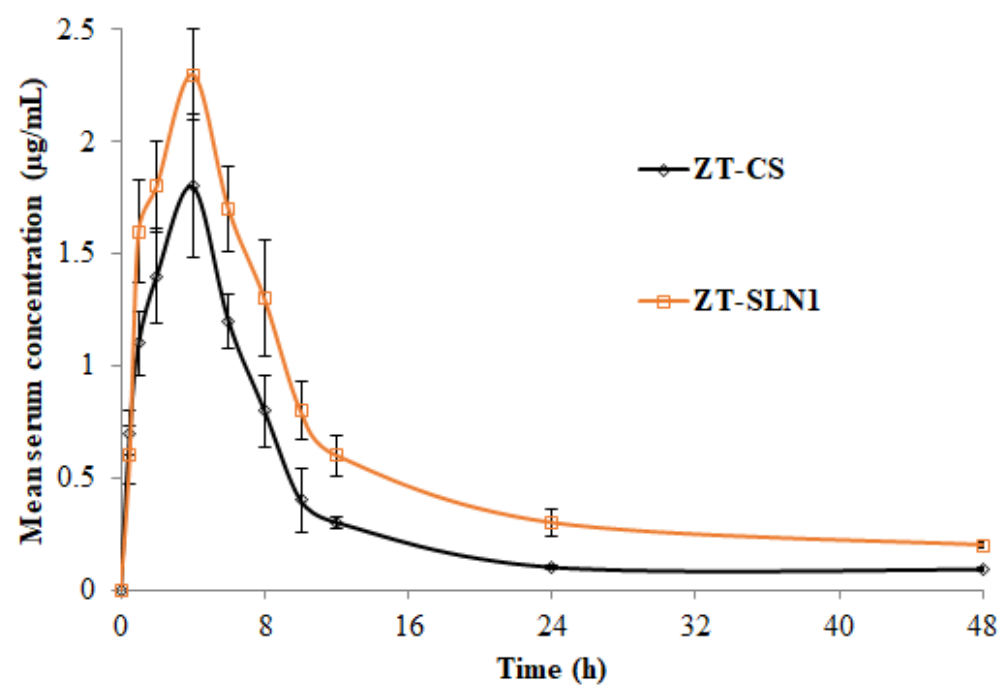

Figure 6: Mean serum concentration - time profiles of zotepine from ZT-CS and optimized ZT-SLN1 formulation after oral administration in rats (mean $\pm S D, n=6$ )

Table 5: Pharmacokinetic parameters of zotepine from zotepine coarse suspension (ZT-CS) and zotepine solid lipid nanoparticle (ZT-SLN1) formulations after oral administration in rats (mean $\pm S D, n=6$ )

\begin{tabular}{|c|c|c|}
\hline Parameter & ZT-CS & ZT-SLN1 \\
\hline$C_{\text {max }}(\mu \mathrm{g} / \mathrm{mL})$ & $1.8 \pm 0.3$ & $2.3 \pm 0.2^{\circ}$ \\
\hline $\mathrm{T}_{\text {max }}(\mathrm{h})$ & 4 & 4 \\
\hline $\mathrm{AUC}_{\text {total }}(\mu \mathrm{g} / \mathrm{mL}) . \mathrm{h}$ & $18.2 \pm 1.3$ & $37.1 \pm 2.2^{*}$ \\
\hline $\mathrm{t}_{1 / 2}(\mathrm{~h})$ & $18.6 \pm 1.2$ & $18.5 \pm 2.1$ \\
\hline MRT $(\mathrm{h})$ & $17.7 \pm 2.6$ & $23.8 \pm 1.8^{*}$ \\
\hline
\end{tabular}

*indicates statistically significant at $\mathrm{p}<0.05$ in comparison with ZT-CS.

ZT loaded solid lipid nanoparticles were successfully developed for the improved oral delivery. The ZT-SLNs were prepared with well-known reported method and characterized for an optimal system. DSC and XRD studies revealed the conversion to amorphous form of ZT in SLN formulation. In vitro and ex vivo permeation studies confirms the sustained release of the ZT from SLN formulation comparison with coarse suspension as control. ZT-SLN formulation stable for two months at storage conditions. Pharmacokinetic study in male Wistar rats revealed that there was 2.0-fold improvement for SLN when compared to coarse suspension. The overall results indicated that the lipid based delivery systems considerably improved the bioavailability of ZT.

\section{Disclosure statement}

The authors declare no conflict of interest in this study. 


\section{REFERENCES}

1. Louis L, Harvey W. Predicting oral drug absorption: Mini Review on physiologically- based pharmacokinetic models. Pharmaceutics. 2017; 9(4): 41.

2. Narendar D, Kishan V. Candesartan cilexetil nanoparticles for improved oral bioavailability. Ther Deli. 2017; 8(2): 79-88.

3. Zhang L, Wang S, Zhang M, Sun J. Nanocarriers for oral drug delivery. J Drug Target. 2o13; 21: 515-527.

4. Arun B, Narendar D. Enhancement of solubility and dissolution rate of trandolapril sustained release matrix tablets by liquisolid compact approach. Asian J Pharma. 2015; 9 (4): 290-297.

5. Mei L, Haonan X, Jingzheng J, et al. Liquisolid technique and its applications in pharmaceutics. Asian J Pharm Sci. 2017; 12: 115-123.

6. Nagaraj K, Narendar D, Kishan V. Development of olmesartan medoxomil optimized nanosuspension using Box-Behnken design to improve oral bioavailability. Drug Dev Ind Pharm. 2017; 43(7): 1186-1196.

7. Yanbin H, Wei-Guo D. Fundamental aspects of solid dispersion technology for poorly soluble drugs. Acta Pharm Sin B. 2014; 4(1): 18-25.

8. Palem CR, Narendar D, Satyanarayana G, Varsha BP. Development and optimization of Atorvastatin calcium-cyclodextrin inclusion complexed oral disintegrating tablets for enhancement of solubility, dissolution, pharmacokinetic and pharmacodynamic activity by central composite design. Int J Pharm Sci Nanotech. 2016; 9(2): 1-11.

9. Serajuddin AT. Salt formation to improve drug solubility. Adv Drug Deliv. Rev. 2oo7; 59(7): 603-616.

10. William NC, Christopher JHP. Lipophilic prodrugs designed for intestinal lymphatic transport. Adv Drug Deliv Rev. 1996;19: 149-169.

11. Natalie LT, William NC, Christopher JHP. Lipid- based delivery systems and intestinal lymphatic drug transport: A mechanistic update. Adv Drug Deliv Rev. 2oo8; 60: 702-716.

12. Müller RH, Mäder K, Gohla S. Solid lipid nanoparticles (SLN) for controlled drug delivery - a review of the state of the art. Eur J Pharm Biopharm. 2ooo; 5O(1): 161-77.

13. Mehnert W, Mäder K. Solid lipid nanoparticles. production, characterization and applications. Adv Drug Deliv Rev. 2012; (64): 83-101.

14. Wenyu W, Xiuli Z, Haiyang H, Dawei C, Jianchun G, Yihui D, Jin S. Galactosylated solid lipid nanoparticles with cucurbitacin B improves the liver targetability. Drug Deliv 2010; 17(3): 114-122.

15. Tatke A, Dudhipala N, Janga KY, Balguri SP, Avula B, Monica MJ, Majumdar S. In situ gel of triamcinolone acetonide-loaded solid lipid nanoparticles for improved topical ocular delivery: tear kinetics and ocular disposition studies. Nanomaterials (Basel). 2018; 27: 9(1).

16. Ben G. Zotepine: a clinical review. Expert Opin Drug Metab Toxicol. 2oo9; 5(2): 81-86.

17. Tanaka O, Kondo T, Otani K, Yasui N, Tokinaga N, Kaneko S. Single oral dose kinetics of zotepine and its relationship to prolactin response and side effects. Ther Drug Moni 1998; 20(1): 117-119.

18. Tanaka O. Pharmacokinetics of zotepine and various factors affecting that of zotepine. Nihon Shinkei Seishin Yakurigaku Zasshi. 1996; 16(2): 49-52. 
19. Pailla SR, Talluri S, Rangaraj N, Ramavath R, Challa VS, Doijad N, Sampathi S. Intranasal zotepine nanosuspension: intended for improved brain distribution in rats. Daru. 2019, 29. doi: 10.1007/s40199-019-00281-4.

20. Dalvadi H, Patel N, Parmar K. Systematic development of design of experiments (DoE) optimised self-microemulsifying drug delivery system of zotepine. J Microencapsul. 2017; 34: 308-18.

21. Mangesh B, Prashant U, Ashwini M. Formulation and characterization of solid lipid nanoparticles for an anti-retroviral drug darunavir. App Nanosci. 2017; 7(1-2): 47-57.

22. Narendar D, Kishan V. Improved anti-hyperlipidemic activity of Rosuvastatin Calcium via lipid nanoparticles: pharmacokinetic and pharmacodynamic evaluation. Euro J Pharm Biopharm. 2017;110 (1): 47-57.

23. Narendar D, Kishan V. Candesartan cilexetil loaded solid lipid nanoparticles for oral delivery: characterization, pharmacokinetic and pharmacodynamic evaluation. Drug Deliv. 2014;23(2): 395-404.

24. Narendar D, Kishan V. Pharmacokinetic and pharmacodynamic studies of nisoldipineloaded solid lipid nanoparticles developed by central composite design. Drug Dev Ind Pharm. 2015; 41(12): 1968-77.

25. Arun B, Narendar D, Kishan V. Development of olmesartan medoxomil lipid-based nanoparticles and nanosuspension: preparation, characterization and comparative pharmacokinetic evaluation. Artif cells. Nanomed. Biotechnol. 2017, 46(1), 126-137.

26. Mai MG, Sana MM, Marwa AS. Hexagonal liquid crystalline nanodispersions proven superiority for enhanced oral delivery of rosuvastatin: in vitro characterization and in vivo pharmacokinetic study. J Pharma Sci. 2017; 106: 3103-3112.

27. Mohammad M, Hadi V, Parvin ZM. Bortezomib-loaded solid lipid nanoparticles: preparation, characterization, and intestinal permeability investigation. Drug Dev Ind Pharm. 2018; 44(10): 1598-1605.

28. Narendar D, Riyaz PMD, Ahmed AY, Nagaraj B. Effect of lipid and edge activator concentration on development of Aceclofenac loaded transfersomes gel for transdermal application: in vitro and ex vivo skin permeation. Drug Dev Ind Pharm. 2o20; 46(8):1334-1344.

29. Butreddy A, Dudhipala N, Janga KY, Rajendra PG. Lyophilization of small-molecule injectables: an industry perspective on formulation development, process optimization, scale-up challenges, and drug product quality attributes. AAPS PharmSciTech. 2o2o; 21: 252.

30. Thirupathi G, Swetha E, Narendar D. Role of isradipine loaded solid lipid nanoparticles in the pharmacodynamic effect of isradipine in rats. Drug Res. 2017; 67(03): 163-169.

31. Narendar D, Karthik YJ. Lipid nanoparticles of zaleplon for improved oral delivery by BoxBehnken design: Optimization, in vitro and in vivo evaluation. Drug Dev Ind Pharm. 2017; 43(7):1205-1214.

32. Narendar D, Karthik YJ, Thirupathi G. Comparative study of nisoldipine-loaded nanostructured lipid carriers and solid lipid nanoparticles for oral delivery: preparation, characterization, permeation and pharmacokinetic evaluation. Artificial cells. Nanomed Biotech. 2018; 46(S2): 616-625.

33. Shi L, Li Z, Yu L, Jia H, Zheng L. Effects of surfactants and lipids on the preparation of solid lipid nanoparticles using double emulsion method. J Disp Sci Techno. 2o11; 32(2): 254-259.

34. Xin, W. L.; Xiao, H. L.; Li, Q. Z.; ; Hong, Z.M. Preparation, characterization, and in vitro release of chloramphenicol loaded solid lipid nanoparticles. J Disp Sci Techno. 20o8, 29(9), 1214-1221. 
35. Pitta S, Dudhipala N, Narala A, Veerabrahma K. Development and evaluation of zolmitriptan transfersomes by Box-Behnken design for improved bioavailability by nasal delivery. Drug Dev Ind Pharm. 2018; 44(3):484-492.

36. Arik D, Amnan H. The effect of different lipid based formulations on the oral absorption of lipophilic drugs: The ability of in vitro lipolysis and consecutive ex vivo intestinal permeability data to predict in vivo bioavailability in rats. Euro J Pharm Biopharm. 2007; 67: 96-105.

37. Narendar D. A comprehensive review on solid lipid nanoparticles as delivery vehicle for enhanced pharmacokinetic and pharmacodynamic activity of poorly soluble drugs. Int J Pharm Sci Nanotech. 2019; 12(2): 4421-4440.

38. Dudhipala N, Puchchakayala G. Capecitabine lipid nanoparticles for anti-colon cancer activity in 1, 2-dimethylhydrazine-induced colon cancer: Preparation, cytotoxic, pharmacokinetic, and pathological evaluation. Drug Dev Ind Pharm. 2018; 44(10): 1572-1582.

39. Narendar D, Thirupathi G. Neuroprotective effect of ropinirole lipid nanoparticles enriched hydrogel for Parkinson's disease: in vitro, ex vivo, pharmacokinetic and pharmacodynamic evaluation. 2020. Pharmaceutics: 12(5), E448. doi: 10.3390/pharmaceutics12050448

40. Tirumalesh C, Suram D, Dudhipala N, Banala N. Enhanced Pharmacokinetic Activity of Zotepine via Nanostructured Lipid Carrier System in Wistar Rats for Oral Application. Pharm Nanotechnol. 2020; 8(2):148-160.

41. Nagaraj B, Tirumalesh C, Dinesh S, Narendar D. Zotepine loaded lipid nanoparticles for oral delivery: development, characterization, and in vivo pharmacokinetic studies. Fut $J$ Pharm Sci. 2020; 6: 1-11.

42. Ahmed AAY, Narendar D, Mujumdar S. Ciprofloxacin loaded nanostructured lipid carriers incorporated into in-situ gels to improve management of bacterial endophthalmitis. Pharmaceutics. 2020; $12(6): 572$.

43. Krishna MV, Kumar BV, Dudhipala N. In-situ intestinal absorption and pharmacokinetic investigations of carvedilol loaded supersaturated self-emulsifying drug system. Pharm nanotech. 2020; 8(3):207-224.

44. Dudhipala N, Ay AA. Amelioration of ketoconazole in lipid nanoparticles for enhanced antifungal activity and bioavailability through oral administration for management of fungal infections. Chem Phy Lipids. 2020; 104953.

45. Suvarna G, Narender D, Kishan V. Preparation, characterization and in vivo evaluation of rosuvastatin calcium loaded solid lipid nanoparticles. Int J Pharm Sci Nanotech. 2015; 8(1): 2779-2785.

46. Sandeep V, Narendar D, Arjun N, Kishan V. Lacidipine loaded solid lipid nanoparticles for oral delivery: preparation, characterization and in vivo evaluation. Int J Pharm Sci. Nanotech. 2016; 9(6):3524-30.

47. Gondrala UK, Dudhipala N, Kishan V. Preparation, characterization and in vivo evaluation of felodipine solid-lipid nanoparticles for improved oral bioavailability. Int J Pharm Sci Nanotech. 2015; 8 (4): 2995-3002. 